\title{
DEVELOPING COUNTRIES CORNER
}

\section{A LITERARY CAFE IN YAOUNDÉ, CAMEROUN}

In 2015, the University of Padua, specifically the Environmental Engineering research group, developed a bottom up cultural project in Yaoundé, Cameroon, to raise awareness in environmental topics, particularly solid waste management, involving local people of different cultural levels.

\section{The background}

Yaoundé counts more than 4.000 .000 inhabitants with a rate of population growth in the range of $7-10 \%$. Nearly $80 \%$ of the urban population lives in informal settlements occupying $72 \%$ of the urban space. $46 \%$ of the population has no access to improved water sources and uses latrines as wastewater devices, mainly located close to water wells (UN-Habitat, 2015). The weekly production of municipal waste in the town has been calculated in about $1200 \mathrm{t}$.

Less than $50 \%$ of waste produced is collected by the commissioned company HYSACAM, and only along the main roads, while in the poor neighborhood (shanty towns) the wastes are abandoned on the road or in water channels and often burned in the open air (Figure 1). No formal recycling is planned and only $5 \%$ of waste is informally recycled by private citizens. Awareness of the people with regard to environmental and health issues linked to wastewater and solid waste management should be urgently improved, although no educational material is available, particularly for the unschooled people.

Within this framework, a decision was reached to design a venue open to the public of all ages and levels of education, where they can find more or less detailed information (video, books, journals, etc.) relating to environmental issues, can connect to the internet to browse international related platforms, and in which seminars on specific topics can be organized to encourage debate and raise awareness.

\section{Specific aims}

To meet the main objective, the project has been designed to include a series of specific aims, based on the model of the literary cafe, seeking to promote culture particularly on environmental topics. Unlike traditional literary cafés, the venue in Yaoundé likes to welcome common people rather than intellectuals, since the priority is to contribute towards solving the daily problems caused by poor sanitation in town. Local and visiting experts are invited to promote dialogue.

The physical space has been created to host creative interactions and conversations, outreach and training seminars: to organise meetings to facilitate the discussion between groups of people on specific topics; to promote the link between different networks; to support young people or students in improving their knowledge.

The literary café was initially financed by a series of different institutions, but in order to reach the economic sustainability, after a start up phase, the organizers set up a business activities.

\section{Designing the project}

The project provides for the realization of an appropriate cultural space in which to collect and boost interest on environmental topics, promoting awareness and disseminating the best practice with regard to daily solid and liquid
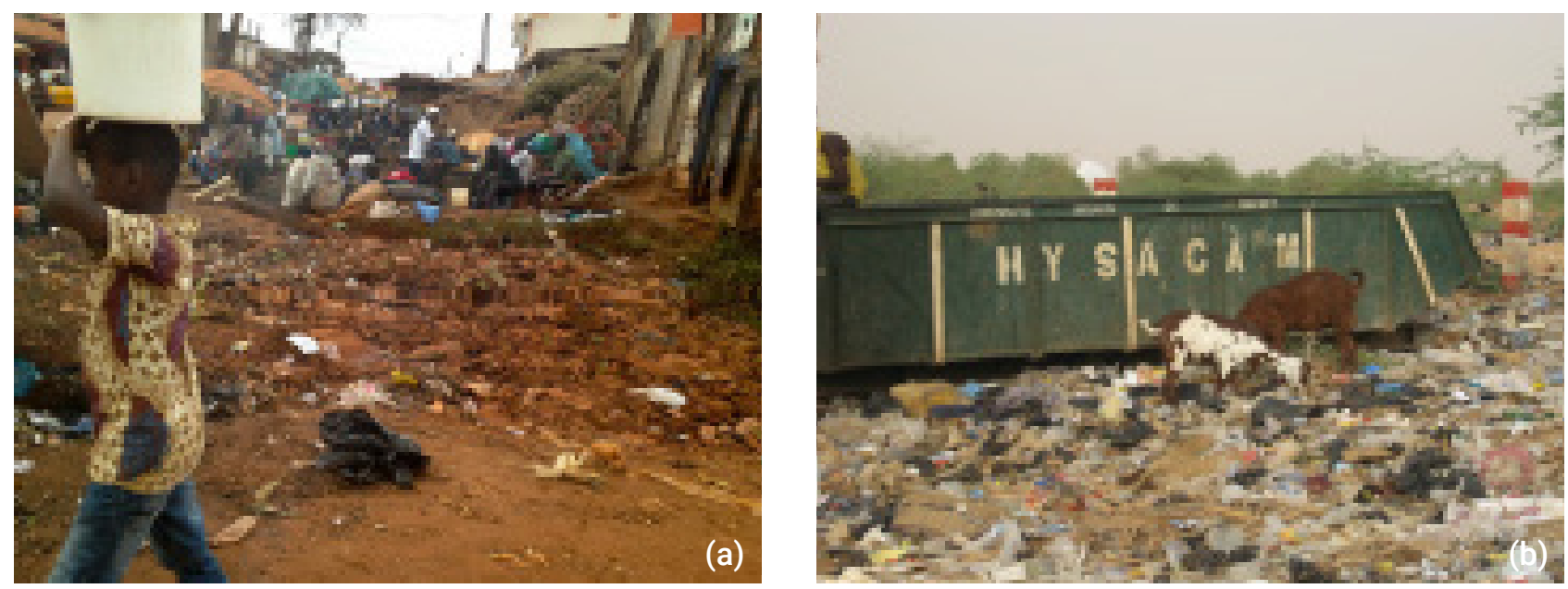

FIGURE 1: Waste in Yaoundé: a) in a poor neighborhood; b) along a main road in town.

Managing editor:

Maria Cristina Lavagnolo

email: mariacristina.lavagnolo@unipd.it
Pe Detritus / Volume $01-2018$ / pages I-III https://doi.org/10.26403/detritus/2018.25 (C) 2018 Cisa Publisher 
waste management.

Basing on a preliminary survey, the space should be integrated into the local social context in order to involve the different sections of the population (housewives, schoolchildren, university students, educators, researchers) and organized in such a way as to implement the different levels of awareness.

To promote the success of this venture, a local no-profit organization, TAM TAM Mobile (Figure 2), an apolitical company founded in 1997 by the director Simon Pierre Etonga with the specific ethic aims of contributing to environmental protection, has been involved.

TAM TAM Mobile organizes informal door-to-door solid waste collection in the poorest neighborhoods where Hysacam is not present, employing approx. 20 young operators, using hand barrows when feasible, since many houses are not accessible.

The activity is economically supported by those residents who are willing to pay small amounts for the service. Waste is collected at home and then disposed in the nearest Hysacam container along the main road: 20000 people are served in 10 different slums and $960 \mathrm{t} / \mathrm{y}$ of waste is collected. This practice is combined with an awareness-raising activity addressed to the population and local authorities on the potential risks to man and the environment linked to poor waste management.

\section{The literary cafe}

A cultural and convivial space has been designed as a pole of interest for the citizenship, a reference point for educational institutions and a meeting place and debate on environmental issues for the population. The space has been identified within a popular district through the collaboration of TAM TAM Mobile (Figure 3).

The project was then finalized, obtaining the necessary authorizations, assessing the restoration works and selecting the furniture in such a way that the space created can provide different types of services: bar, restaurant and catering, services that can attract and welcome diversified people and that, after an initial start-up phase, facilitate economic autonomy; consultation of brochures, books and magazines at different levels of in-depth analysis of envi-

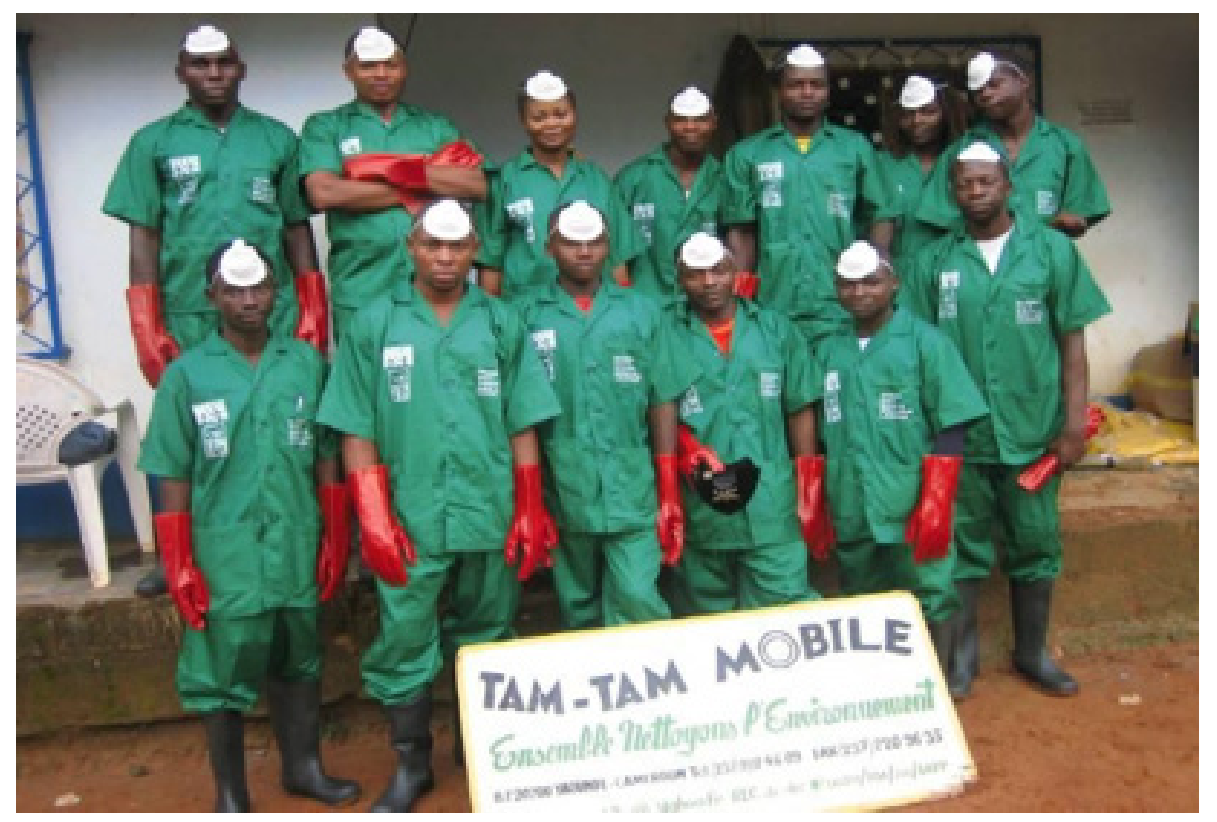

FIGURE 2: The TAM Tam Mobile team, Yaoundé.
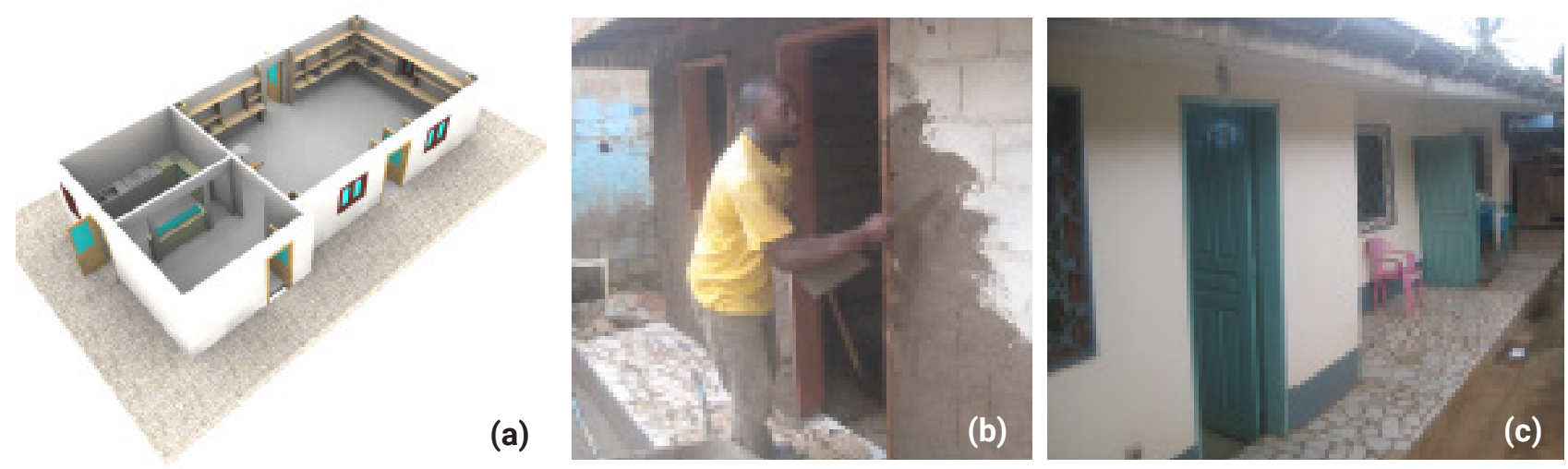

FIGURE 3: Different phases of the literary cafe: a) the project (courtesy of arch. Rosalba Giani), b) the restoration works, c) the realisation 
ronmental issues; use of computers equipped with an internet connection in order to carry out research in the relevant sector, consult online journals, join and keep in touch with thematic networks; organization of small events like public meetings to inform, seminars and discussion groups, projections of documentaries and other material relating to environmental issues, laboratory activities of sensitization.

\section{In action}

The literary cafe opened at the end of 2015 and is managed by TAM TAM Mobile. From that moment many activities have been carried out (Figure 4).

The activities were particulary based on:

- Awareness and information campaigns on environmental issues for all

Seminars and training on specific topics (municipal garbage, WEEE recycling, computer laboratory); production and distribution of informative material explaining the actions needed to prevent health risks due to poor sanitary conditions due to the huge presence of abandoned waste.

- Education, training for women and young people in situ- ations of vulnerability

To meet the most basic needs, laboratories have been set up as awareness campaigns against gender-based violence and to promote women in the business world. At the end of each event, a technical report is produced and distributed ex post to the participants highlighting the principal results obtained during the discussion that follows.

\section{Partners}

The project has been funded by the following organisations:

- Regione del Veneto

- University of Padova

- Rotary Club of Padova

- Faber Libertatis

- IWWG-International Waste Working Group

Maria Cristina Lavagnolo *, Silvia Failli University of Padova, Italy * email: mariacristina.lavagnolo@unipd.it

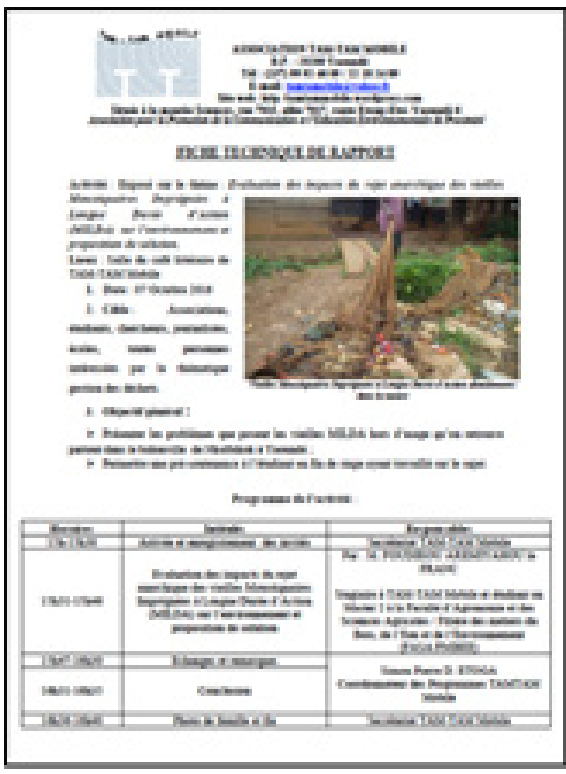

(a)

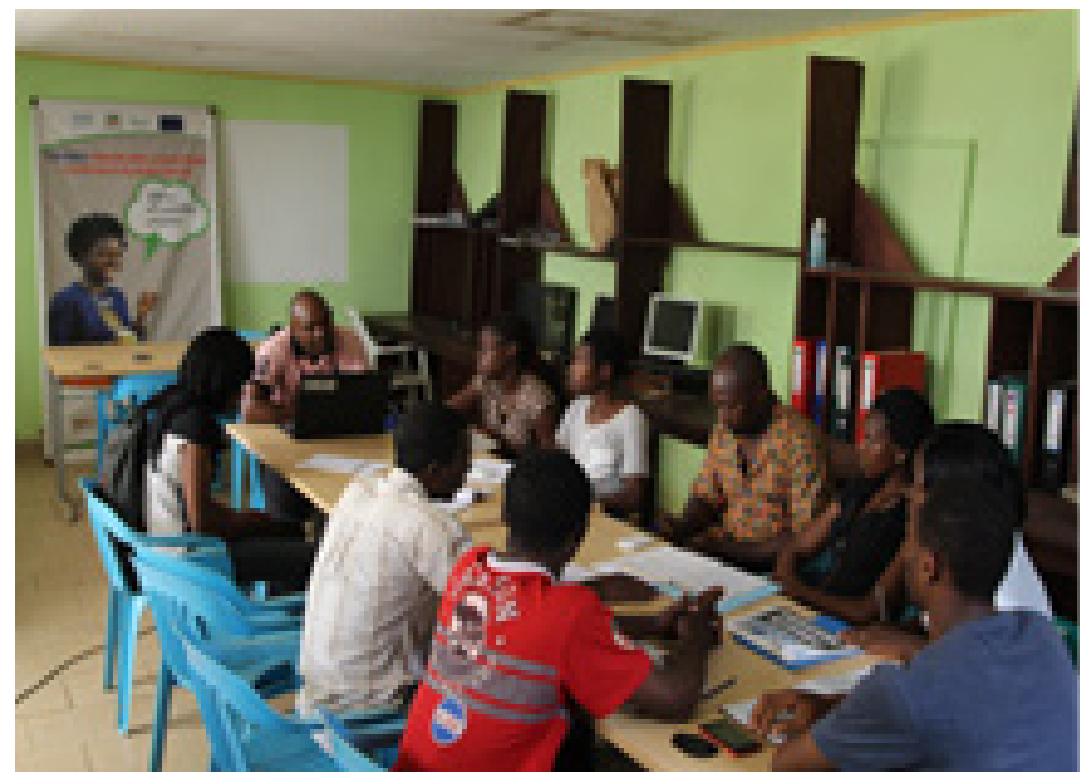

(b)

FIGURE 4: Event organised in the literary café, (a) and typical final report used as follow up document (b). 